\title{
Reduced Graphene Oxide Incorporated GelMA Hydrogel Promotes Angiogenesis For Wound Healing Applications
}

This article was published in the following Dove Press journal: International Journal of Nanomedicine

\author{
Syed Raza ur Rehman ${ }^{1,2}$ \\ Robin Augustine ${ }^{1,2}$ \\ Alap Ali Zahid (D) ${ }^{1,2}$ \\ Rashid Ahmed ${ }^{1,2}$ \\ Muhammad Tariq (D) $^{3}$ \\ Anwarul Hasan (iD) ${ }^{1,2}$ \\ 'Department of Mechanical and Industrial \\ Engineering, College of Engineering, \\ Qatar University, Doha 27I3, Qatar; \\ ${ }^{2}$ Biomedical Research Center, Qatar \\ University, Doha 2713, Qatar; \\ ${ }^{3}$ Department of Biotechnology, Faculty of \\ Science, Mirpur University of Science and \\ Technology, Mirpur 10250, AJK, Pakistan
}

Correspondence: Anwarul Hasan

Department of Mechanical and Industrial

Engineering, College of Engineering,

Qatar University, Doha 2713, Qatar

Tel +97470569169

Email hasan.anwarul.mit@gmail.com
Purpose: Non-healing or slow healing chronic wounds are among serious complications of diabetes that eventually result in amputation of limbs and increased morbidities and mortalities. Chronic diabetic wounds show reduced blood vessel formation (lack of angiogenesis), inadequate cell proliferation and poor cell migration near wounds. In this paper, we report the development of a hydrogel-based novel wound dressing material loaded with reduced graphene oxide ( $\mathrm{rGO}$ ) to promote cell proliferation, cell migration and angiogenesis for wound healing applications.

Methods: Gelatin-methacryloyl (GelMA) based hydrogels loaded with different concentrations of rGO were fabricated by UV crosslinking. Morphological and physical characterizations (porosity, degradation, and swelling) of rGO incorporated GelMA hydrogel was performed. In vitro cell proliferation, cell viability and cell migration potential of the hydrogels were analyzed by MTT assay, live/dead staining, and wound healing scratch assay respectively. Finally, in vivo chicken embryo angiogenesis (CEO) testing was performed to evaluate the angiogenic potential of the prepared hydrogel.

Results: The experimental results showed that the developed hydrogel possessed enough porosity and exudate-absorbing capacity. The biocompatibility of prepared hydrogel on three different cell lines (3T3 fibroblasts, EA.hy926 endothelial cells, and HaCaT keratinocytes) was confirmed by in vitro cell culture studies (live/dead assay). The GelMA hydrogel containing $0.002 \% \mathrm{w} / \mathrm{w}$ rGO considerably increased the proliferation and migration of cells as evident from MTT assay and wound healing scratch assay. Furthermore, rGO impregnated GelMA hydrogel significantly enhanced the angiogenesis in the chick embryo model.

Conclusion: The positive effect of $0.002 \% \mathrm{w} / \mathrm{w}$ rGO impregnated GelMA hydrogels on angiogenesis, cell migration and cell proliferation suggests that these formulations could be used as a functional wound healing material for the healing of chronic wounds.

Keywords: GelMA hydrogel, reduced graphene oxide, nanocomposite hydrogel, angiogenesis, wound healing

\section{Introduction}

Delayed wound healing is a common complication of diabetes mellitus affecting about $15 \%$ of the diabetes patients which can often result in the development of diabetic foot ulcers (DFUs). ${ }^{1}$ This usually leads to repeated hospitalizations, higher healthcare cost, poor quality of life and in some extreme cases amputation of the affected organ or limb. ${ }^{2}$ The major reasons for the persistent delay in the wound healing are chronic inflammation, disturbed growth factor secretion, recurrent 
microbial infections and disrupted angiogenesis. ${ }^{3-7}$ Another reason related to the delay in healing process is the decrease in the proliferation, migration, and relocation of native cells including fibroblasts and keratinocytes which are needed for the development of a functional skin. ${ }^{8}$ In addition, other factors such as excessive secretion of tissue degrading enzymes, prolonged microbial/bacterial infections and absence of vascularization play a prominent role in the occurrence of non-healing wounds. Currently, there is no fully effective therapy for managing diabetic wounds. Some potential solutions that were proposed and are being investigated by researchers include the use of tissue engineering techniques, improving the angiogenic activity within damaged tissues, and supplying growth factors necessary for wound healing. ${ }^{9-11}$ Current techniques for enhancing the formation of vascular networks can be categorized into three types: microengineering techniques, ${ }^{12}$ cell-based techniques, ${ }^{13-15}$ and biochemical approaches. ${ }^{16}$ Microengineering techniques involve the use of microfabrication techniques for the formation of new blood vessels within the polymeric scaffolds. Whereas cell-based techniques use the progenitor or mature endothelial cells, co-cultured within the polymeric scaffolds for promoting angiogenic activity, while biochemical techniques involve the incorporation of the small bioactive molecules or growth factors within scaffolds for promoting formation of the blood vessels. However, there are some limitations in all of these approaches. For example, the micro-engineering technique suffers from problems of host-implant integrations, and cell-seeded skin substitutes lack proper control over the integration of the host cells and differentiation of the seeded cells. ${ }^{17}$ However, biochemical approaches suffer due to the short half-life of the growth factors in physiological conditions. ${ }^{17}$ Therefore, effective strategies are needed to facilitate the regeneration of damaged tissues and reverse the factors that hamper angiogenic activity which is considered as one of the major requirement for promoting wound healing.

Several wound dressing materials have been used from ancient times to provide protection to the wounds from various types of pathogens by acting as a barrier from the outer environment. $^{4,6,18}$ However, the advanced and functional wound coverage materials are expected to have remarkable wound healing properties. ${ }^{19}$ For instance, it's a common observation that wounds generally heal faster in the presence of moist and porous dressings. ${ }^{20,21}$ An ideal wound dressing should be able to manage excess exudates produced by the wounds. ${ }^{22}$ Hydrogels are cross-linkable synthetic or natural polymers that can absorb a large amount of water. ${ }^{23}$ Moreover, hydrogels mimic the properties of natural extracellular matrix (ECM) as well as control the release of active ingredients to the tissues. ${ }^{24,25}$ They have been widely applied in numerous biomedical applications such as controlled drug delivery systems, regenerative medicine, and tissue engineering. ${ }^{26-35}$ Their excellent hydrophilic property due to the existence of hydrophilic groups, such as hydroxyl, carboxyl, amino, and amido groups in the polymer chains, makes them more feasible for wound healing applications. They can be fabricated as highly porous, soft and flexible structures which permeable to water vapors. Furthermore, hydrogels can securely cover the wounds and prevent the infection by microorganisms. Liang et al developed a conductive injectable nanocomposite hydrogel with photothermal antibacterial activity and sustained drug release to promote full-thickness skin regeneration. ${ }^{36}$ Injectable hydrogels were also fabricated with multifunctional properties for localized drug delivery in wounds. ${ }^{37-39}$ Majumder et al have developed a multicompartment hydrogel material that allows the time-independent release of small molecules. ${ }^{39}$ However, the selection of right biopolymers and the agents that can facilitate angiogenic activity in the healing process is highly important.

Gelatin Methacryloyl (GelMA) hydrogels are prepared from porcine-derived gelatin crosslinked with methacrylic anhydride. ${ }^{40}$ GelMA hydrogels have highly tunable physical characteristics and suitable biological properties. Some cell attachment and matrix metalloproteinase responsive peptide motifs are present in GelMA hydrogel, which allow the cells to proliferate and spread well in the scaffold. Due to this property, GelMA based hydrogel closely resembles some important properties of natural ECM. ${ }^{41}$ GelMA hydrogels can be crosslinked using relatively a very low concentration of photo-initiator within a minute or even seconds. ${ }^{42}$ Additionally, GelMA hydrogels are less expensive, non-immunogenic and possess excellent biocompatibility due to the presence of gelatin. ${ }^{43}$ Moreover, the degradation, mechanical and biological properties of GelMA hydrogel can easily be modified by varying the concentration of GelMA prepolymer, methacrylation degree or photo-polymerization time. ${ }^{44,45}$

Nanomaterials are being loaded in polymeric wound coverage matrices to provide antibacterial property, enhancing cell proliferation, improving the angiogenic potential and facilitating rapid wound healing. ${ }^{46,47}$ Recently, graphene, graphene oxide (GO) and reduced graphene oxide (rGO) nanostructures have attracted 
great deal of attention as an inorganic additive in biopolymers for the development of novel composite biomaterials. ${ }^{48}$ Their excellent properties such as the ability to promote angiogenesis, ${ }^{9}$ relatively high biocompatibility, ${ }^{49}$ unique chemical and physical properties. ${ }^{48}$ make them one of the most favorable materials for many biomedical applications. ${ }^{50}$ Graphene derivatives have significant potential in nanomedicines, ${ }^{51,52}$ anticancer drug delivery systems, ${ }^{53}$ biological sensors, ${ }^{54}$ as cancer biomarkers, ${ }^{40}$ as catalysts, ${ }^{51,55}$ and as better antibacterial agents. ${ }^{56}$ Furthermore, a recent work reported that graphene oxide (GO) and reduced graphene oxide (rGO) display a considerable angiogenic activity in a dose-dependent manner. ${ }^{9}$ It was reported that the rGO has the ability to increase the concentration of reactive oxygen species (ROS) ${ }^{9}$ in biological systems and can play an important role in angiogenesis. ${ }^{57}$ ROS can also act as a signalling molecule in many aspects of growth factor-mediated physiological responses such as cell proliferation and wound healing. ${ }^{57}$ Earlier studies demonstrated that incorporation of nanomaterials that can generate ROS in polymeric biomaterials can enhance cell proliferation and blood vessel formation. ${ }^{58}$ Reduced graphene oxide induces angiogenesis by stimulating cell proliferation and migration. ${ }^{9,59}$ Based on this observation, we hypothesized that developing rGO impregnated GelMa hydrogel could be a useful strategy for accelerating the healing of chronic wounds.

In this work, we developed a novel rGO impregnated GelMa hydrogel as a functional dressing material to promote cell proliferation, cell migration and angiogenesis for achieving rapid wound healing. GelMA hydrogel with varying concentration of $\mathrm{rGO}$ was synthesized to get an optimum concentration of rGO to enhance angiogenesis. The developed nanocomposite hydrogel was characterized using various physical characterization methods. The biocompatibility of GelMA hydrogel and nanocomposite hydrogels was tested on three different cell lines. The ability of the nanocomposite hydrogel to promote cell proliferation and cell migration were evaluated in-vitro. Finally, the potential of the nanocomposite hydrogel to promote angiogenesis was demonstrated through in vivo angiogenesis assay. To the best of our knowledge, this is the first time that is being reported the use of $\mathrm{rGO}$ within GelMa hydrogel for wound healing applications. We believe that this work will help in advancing the management of trauma care where promoting angiogenesis is crucial, particularly in chronic diabetic wounds.

\section{Materials And Methods Materials}

Gelatin (Type A) from porcine skin and methacrylic anhydride (MA) were purchased from Sigma-Aldrich. Reduced graphene oxide powder was purchased from Graphitene, UK. The dialysis tubings and Live/Dead cell Imaging kit were purchased from Thermo Fisher Scientific. The phosphate buffer saline (PBS), Dulbecco's modified eagles medium (DMEM), Fetal bovine serum, Penicillin-streptomycin solution, and trypsinEDTA solution were obtained from Gibco, USA. N-Methyl-2pyrrolidone (NMP) was purchased from VWR, USA. The fibroblasts, keratinocytes and endothelial cells were kindly received as a gift from Dr. Su Ryon Shin from Shin Laboratory, Birmingham and Women's Hospital, Cambridge, MA, USA. Biological experiments were performed with the approval of Institutional Biosafety Committee, Qatar university (QU-IBC-2018/053).

\section{Preparation Of Nanocomposite GelMA Hydrogel}

Porcine-derived gelatin was dissolved in PBS at $60^{\circ} \mathrm{C}$ and $8 \mathrm{~mL}$ MA was added for every $100 \mathrm{~mL}$ of gelatin solution under vigorous stirring $\left(50^{\circ} \mathrm{C}\right)$ at $0.5 \mathrm{~mL} / \mathrm{min}$ until completely dissolved. The mixture was then reacted for $3 \mathrm{hrs}$. The reaction was stopped by adding $400 \mathrm{~mL}$ of preheated PBS $\left(50^{\circ} \mathrm{C}\right)$. The solution was then dialyzed against distilled water for 1 week at $40^{\circ} \mathrm{C}$ and then freeze-dried to obtain white porous foam-like GelMA prepolymer.

$5 \mathrm{mg}$ of rGO was added in $1 \mathrm{~mL}$ of N-Methyl-2pyrrolidone (NMP) and dispersed very well using ultrasonicator (Model 150VT, Ultrasonic Homogenizer, at 100 $\mathrm{W}$ for $1 \mathrm{hr}$ ). The suspension was then diluted to $0.5 \mu \mathrm{g} /$ $10 \mu \mathrm{l}, 1 \mu \mathrm{g} / 10 \mu \mathrm{l}$, and $2 \mu \mathrm{g} / 10 \mu \mathrm{l}$ and added in $1 \mathrm{ml}$ of PBS to make $0.001 \% \mathrm{w} / \mathrm{w}$ (GrG1), $0.002 \% \mathrm{w} / \mathrm{w}$ (GrG2) and $0.004 \% \mathrm{w} / \mathrm{w}$ (GrG4) nanocomposite hydrogels respectively. The required amount of freeze-dried GelMA polymer and photoinitiator was mixed with PBS containing rGO separately for each set and vortexed until the GelMA polymer dissolved completely. The concentration of GelMA prepolymer and photoinitiator (Irgacure 2959) in the nanocomposites were maintained as $5 \% \mathrm{w} / \mathrm{v}$ and $0.5 \% \mathrm{w} / \mathrm{w}$, respectively. The prepared solutions $(50 \mu \mathrm{l})$ were placed on round glass coverslips and exposed to UV light $\left(500 \mathrm{~nm}, 7 \mathrm{mwWcm}^{-2}\right)$ for 10 seconds for crosslinked as shown in Figure 1. ${ }^{45,60}$ Control GelMA hydrogels without rGO was also prepared. The compositions of 


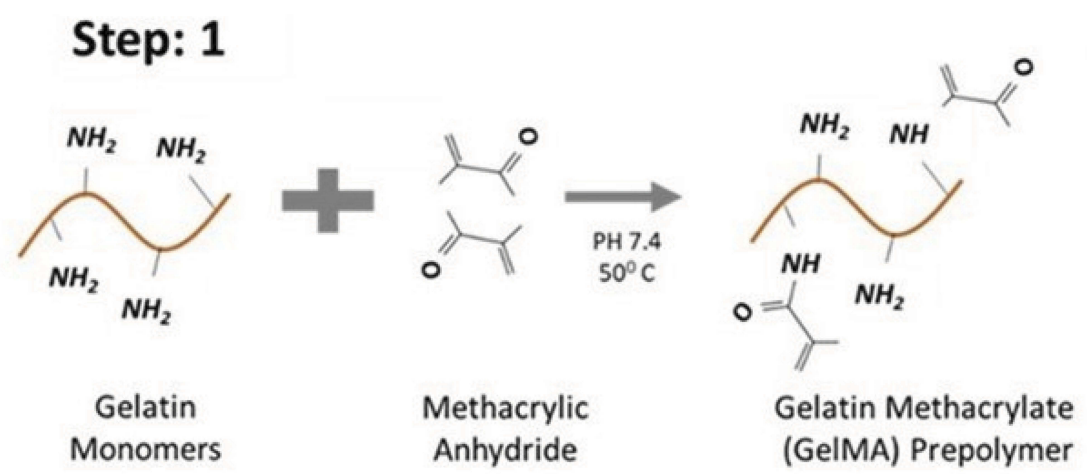

\section{Step: 2}

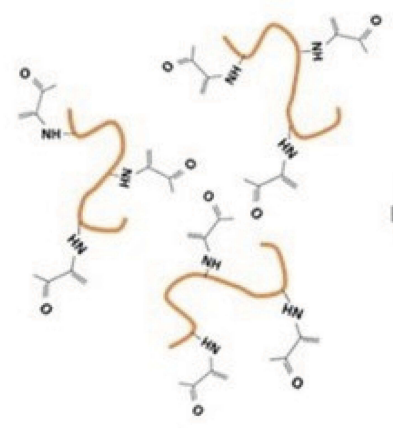

GeIMA Prepolymers

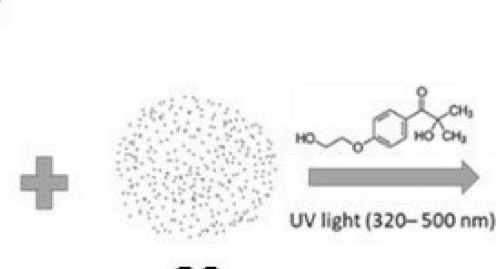

rGO

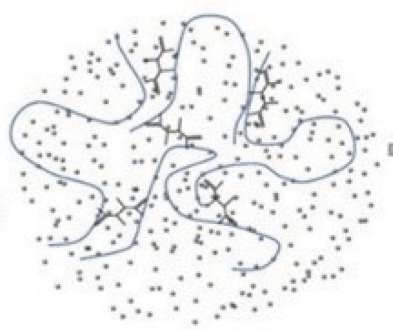

Nanocomposite GeIMA hydrogel

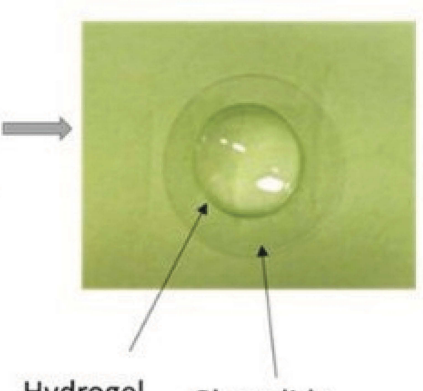

Hydrogel Glass slide

Figure I Schematic representation for the fabrication of nanocomposite GeIMA hydrogel by UV crosslinking method.

GelMA hydrogel of nanocomposite hydrogels are demonstrated in Table 1.

\section{Physical Characterizations} Scanning Electron Microscopy (SEM)

The surface morphology of GelMA and nanocomposite hydrogels were observed using an SEM. Samples were freeze-dried to completely remove the water, affixed on carbon stubs and sputter-coated with a thin layer of gold. The GelMA samples and rGO were observed using a SEM (FEI, Nova NanoSEM, 450 FE-SEM) under an accelerating voltage of $20 \mathrm{kv}$, and a Field Emission Transmission Electron Microscope (HF-3300) respectively.

Table I The Composition Of Hydrogels

\begin{tabular}{|l|l|l|l|}
\hline Samples & Abbreviations & $\begin{array}{l}\text { GelMA } \\
\%(w / v)\end{array}$ & $\begin{array}{l}\text { rGO \% } \\
(\mathbf{w} / \mathbf{w})\end{array}$ \\
\hline GeIMA & GeIMA & 5 & 0 \\
GeIMA 5\% rGO 0.001\% & GrGI & 5 & 0.001 \\
GeIMA 5\% rGO 0.002\% & GrG2 & 5 & 0.002 \\
GelMA 5\% rGO 0.004\% & GrG4 & 5 & 0.004 \\
\hline
\end{tabular}

\section{X-Ray Diffraction Analysis (XRD)}

The XRD pattern of rGO, GelMA and nanocomposite samples (GrG1 and GrG2) were obtained using Empyrean, Malvern Panalytical XRD system (40 kV voltage, $30 \mathrm{~mA}$ current, the scanning rate of $5 \% \mathrm{~min}$ with a step size of $0.032^{\circ}$ ) from $2 \theta$ range from $0^{\circ}$ to $60^{\circ}$.

\section{Degradation Study}

GelMA hydrogel and nanocomposite hydrogels samples were freeze-dried for $24 \mathrm{hrs}$. Initial weight $\left(\mathrm{W}_{0}\right)$ of all the samples were measured and then kept in PBS in an incubator at $37^{\circ} \mathrm{C}$. The PBS was refreshed weekly. The specimens were taken out and dried under vacuum at $50^{\circ} \mathrm{C}$ at predetermined time intervals and final weight $\left(\mathrm{W}_{\mathrm{t}}\right)$ were observed. The degradation rates were calculated using Equation (1).

$$
\text { Degradation rate }(\%)=\frac{W_{0}-W_{t}}{W_{0}} \times 100 \%
$$

\section{Swelling Percentage}

The swelling properties of GelMA hydrogel and nanocomposite hydrogels were investigated by using the 
gravimetric technique. ${ }^{61}$ Firstly, the dried samples were weighed $\left(\mathrm{W}_{\mathrm{dry}}\right)$ and then placed in Petri dishes filled with distilled water (DW). The Petri dishes were then placed in a temperature-controlled water bath at the room temperature. Finally, the samples were taken out at different time intervals and weighed. Before weighing, the samples were rubbed with a filter paper to remove the excess water. The swelling property was then calculated by using Equation (2).

$$
Q(g / g)=\left(W_{w e t}-W_{d r y}\right) / W_{d r y} * 100
$$

Where $W_{d r y}$ is the weight of dry samples before immersed in water and $W_{\text {wet }}$ is the weight of the hydrogel after being submersed in DW.

\section{Release Study}

Release of rGO from nanocomposite GelMA hydrogel was performed in PBS. Each sample of GelMA hydrogel loaded with three different concentrations of rGO (GrG1, GrG2, and GrG4) and blank GelMA hydrogel was kept in Petri dishes containing $1 \mathrm{~mL}$ of $\mathrm{PBS}$ at $37^{\circ} \mathrm{C}$. The average weight of each sample (freeze-dried) was $4 \mathrm{mg}$. Samples were then withdrawn from the Petri dishes after 1 day, 3 days, and 5 days, respectively. Then, the release of rGO from the GelMA hydrogel was detected by UV-Vis spectrophotometer (PerkinElmer Uv Lambda 25) from the absorbance value at $226 \mathrm{~nm}$. Amount of rGO released from hydrogel samples was calculated from a standard plot of rGO suspensions.

\section{Biological Characterizations MTT Assay}

MTT colorimetric assay was used to analyze the proliferation of the cells on GelMA hydrogel and nanocomposite hydrogels after 1, 3 and 5 days of incubation. The endothelial cells (EA.hy926), HaCat keratinocytes, and 3T3 fibroblasts were seeded on the hydrogel in 24 well plates at the density of $50 \times 10^{3}$ cells/well. Before adding the samples to the 24 well plates it was sterilized with the UV irradiation for $20 \mathrm{~min}$. For determining the cell viability, the culture media was changed and then, $50 \mu 1$ of MTT dye was added. After $4 \mathrm{hr}$ incubation, the supernatant was discarded. Then, $200 \mu \mathrm{l}$ of DMSO (dimethyl sulfoxide) solution was added to dissolve the formazan crystals. Finally, $30 \mu 1$ of the solution from each well was transferred into 96 well plates. The absorbance was read at $570 \mathrm{~nm}$ by using Epoch 2 microplate reader. Cell viability was calculated using Equation (3).

$$
\begin{aligned}
\text { Cell proliferation }(\%)= & \underset{\times 100}{(\text { OD Sample } / \text { OD Control })}
\end{aligned}
$$

All the experiments were repeated 3 times and absorbance were measured in triplicates.

\section{Live/Dead Assay}

The cell viability of GelMA hydrogel and nanocomposite hydrogels has been investigated by Live/Dead assay on 3T3 fibroblasts, HaCat keratinocytes, and endothelial cells. The cells were cultured in DMEM medium in the 24 well plates for $24 \mathrm{hrs}$ as explained in the previous section. The hydrogels were then added in each well after changing the medium and kept in the incubator for another $24 \mathrm{hrs}$. Following the manufacturer protocols, cells were then stained with Live/Dead assay kit (Invitrogen R37601). The images were taken using an Olympus fluorescent microscope (Olympus, FV300).

\section{Cell Migration}

Scratch assay was used for the cell migration study by using endothelial (EA.hy926), hacat keratinocyte, and 3T3 fibroblast cells. The cells were seeded on the 12 well plates at the density $50 \times 10^{3}$ cells/well. The culture media was changed after $24 \mathrm{hrs}$ of incubation. When the cells reached $90 \%$ of confluency, a scratch was made using a pipette tip of $100 \mu 1$. Cells were washed with PBS to remove died cells. GelMA hydrogels and nanocomposite hydrogels (GrG1, GrG2, and GrG4) was placed in the wells and allowed to incubate for $24 \mathrm{hrs}$. Time-dependent bright-field images were taken using an Olympus (X53) microscope. Experiments were performed in triplicates. Wound contraction was quantified from the images using Equation (4).

Wound contraction $(\%)=(\mathrm{Wd} 0-\mathrm{Wdt}) / \mathrm{Wd} 0 \times 100$

Whereas $\mathrm{Wd} 0$ is the distance between wound boundaries immediately after wounding procedure and $\mathrm{Wdt}$ is the distance between wound boundaries after time " $t$ " of sample treatment.

\section{CEA Assay}

The chicken embryo angiogenesis (CEA) assay has been performed to determine the effect of GelMA hydrogels and nanocomposite hydrogels on angiogenesis using a reported protocol. ${ }^{60}$ The fertilized chicken eggs (Gallus domesticus) were purchased from Arab Qatari for Poultry Production, Shamal Road, Farm Street, Qatar and 
incubated for 4 days before experiments with a $65 \%$ of relative air humidity and at a temperature of $37^{\circ} \mathrm{C}$. One hour before experiments, a hole was carefully made of $4 \mathrm{~mm}$ diameter and covered with parafilm to prevent dehydration. The eggs were then kept in the incubator for one hour at a static position. A window was carefully created on the top of the eggshell to provide access to the chorioallantoic membrane. Sterile samples of GelMA hydrogel and nanocomposite hydrogels were deposited on the chorioallantoic membrane. The eggs were kept in the incubator for $24 \mathrm{hrs}$ in a static position. The eggs were then observed for angiogenesis and photographs were taken using a Zeiss stereomicroscope (Stemi 508). The thickness and the length of the blood vessel were then quantified using ImageJ software.

\section{Statistical Analysis}

All the experiments were repeated three to four times for each type and data were represented as means and standard deviation (SD). Student's $t$-test and ANOVA was performed between different groups using Minitab statistical software. A p-value less than 0.05 was considered as statistically significant.

\section{Results And Discussions}

In the present work, rGO loaded GelMA hydrogels were developed to assess their effects on the formation of new blood vessels in the chorioallantoic membrane of chick embryo model. The stimulation and acceleration of the angiogenic activity is a major requirement for enhancing the diabetic and burn wound healing. Moreover, proliferation and migration of native cells are hampered by multiple sets of factors in such chronic wounds. It was hypothesized that incorporation of rGO nanoparticles in GelMA hydrogel could trigger the angiogenesis, cell migration and cell proliferation which will enhance the healing process. A brief description of results obtained from the various parameters used to assess the suitability of developed GelMA hydrogels loaded with different concentrations of rGO is described below.

\section{Physical Characterizations}

\section{Surface Morphology}

The images of surface morphology of GelMA hydrogel obtained by Scanning Electron Microscopy (SEM) displayed enormous porous structure both on the surface and in the inner structure. Figure $2 \mathrm{~A}-\mathrm{C}$ shows the surface and inner morphology of GelMA hydrogel. The average pore size of GelMA hydrogel was $50 \mu \mathrm{m}$, as shown in cross-sectional images of Figure $2 \mathrm{~B}$ and $\mathrm{C}$. The porous nature of the hydrogels allows the cells to migrate and proliferate in the hydrogel. Since the amount of rGO inside GelMA hydrogel was very small, the internal structure and pore size of the nanocomposite GelMA hydrogel was not significantly affected by the addition of rGO. The addition of rGO was further confirmed by XRD analysis. Furthermore, the average particle size of rGO was 30-40 $\mathrm{nm}$ from transmission electron microscopy (TEM) (Figure S1) and dynamic light scattering (DLS) analysis (data not shown). Zeta potential of the rGO was $-10.8 \mathrm{mV}$.

\section{X-Ray Diffraction Pattern}

The XRD pattern of the nanocomposite hydrogels was used to determine the nanoparticle formations in GelMA hydrogel networks. The XRD patterns of GelMA hydrogel, rGO, $0.001 \%$ w/w rGO loaded GelMA hydrogel (GrG1) and $0.002 \%$ w/w rGO loaded GelMA hydrogel (GrG2) is given in Figure 2D. The blank hydrogel samples did not exhibit any sharp peak in XRD pattern, with only a broad peak at $2 \theta=32^{\circ}$ attributed to the polymer networks as reported in the study of Lei Zhou et al. ${ }^{62}$ In the case of rGO, two peaks were observed. These two diffraction peaks in the XRD patterns of nanoparticles, at an angle $2 \theta=34^{\circ}$ and $2 \theta=44^{\circ}$ are ascribed to Bragg reflections corresponding to (002) and (100) planes as reported in the study of. ${ }^{63}$ The peak of rGO (002) is present in both GrG1 \& GrG2 hydrogels which were absent in blank GelMA hydrogels pattern as expected. The presence of diffraction patterns of $\mathrm{rGO}$ in the XRD patterns of nanocomposite hydrogels indicates the successful loading of rGO in the GelMA hydrogel.

\section{Degradation Study}

The degradation study of blank GelMA hydrogels and nanocomposite hydrogels were performed to confirm its biodegradability in PBS as shown in Figure 3A. Unlike the unmodified gelatin which dissolves within a few hours in PBS and loose its three-dimensional structure, GelMA could maintain its three-dimensional structure in PBS for 28 days. This also fulfills the requirement of wound healing and several tissue engineering applications. Moreover, the incorporation of rGO in GelMA hydrogel has slightly decreased the degradation rate of the hydrogel. Biodegradability of the prepared GelMA hydrogel has a significant role in wound healing. In general, by hydrolysis and enzymolysis, gelatin can be converted into 

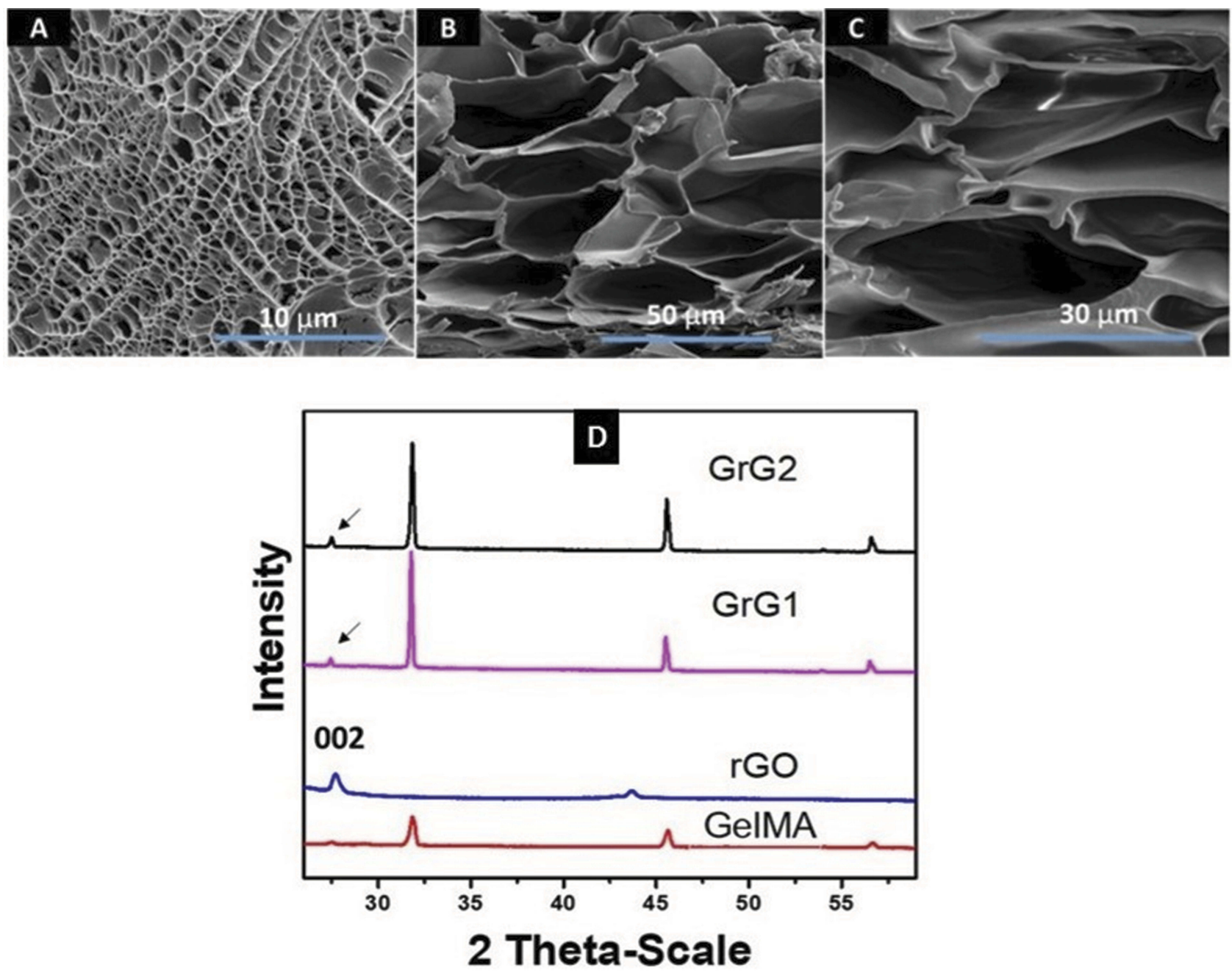

Figure 2 SEM micrograph at 10000X magnification of the surface of (A) GelMA hydrogel, (B) Cross-sectional image of GelMA hydrogel at 2500X, \& (C) Cross-sectional image of GelMA hydrogel at 5000X magnification. (D) XRD curves of blank GelMA hydrogel, rGO nanoparticles, 0.00 I wt\% rGO loaded GelMA hydrogel (GrGI), 0.002 wt $\%$ rGO loaded GelMA hydrogel (GrG2) respectively.

aminophenol and then absorbed by the body which avoids the production of toxic by-products. The biodegradable hydrogel can provide sufficient space for tissue ingrowths, cell growth as well as on cell rearrangement when implanted into the body or during wound healing. ${ }^{64}$

\section{Swelling Properties}

The swelling ability of the GelMA hydrogel and nanocomposite hydrogels were studied to analyze the exudate uptake capacity of the hydrogels. The swelling percentage of both GelMA hydrogel and nanocomposite hydrogels has significantly increased within the first 15 mins of immersion in PBS as shown in Figure 3B. However, the differences in swelling percentage within GelMA hydrogel and nanocomposite hydrogels were not significant. And within 30 mins of immersion, the swelling equilibrium was reached. The incorporation of $\mathrm{rGO}$ has not affected the swelling behavior of the hydrogel.

The ability of the prepared GelMA hydrogel to absorb a large quantity of water (almost 1000\%) within a few minutes of immersion, make them one of the best candidate for developing wound dressings. The water-absorbing ability of the hydrogel provides a moist environment for the cells to grow. Furthermore, it helps to hydrates the wound, re-hydrate eschar and aid in autolytic debridement and also in absorbing excess wound exadute. ${ }^{65}$

\section{Cumulative Release Of rGO}

The release profile of rGO from nanocomposite GelMA hydrogel is given in Figure $3 \mathrm{C}$. The concentration of rGO, measured after each time interval ( 1 day, 3 days, and 5 days) clearly depicts the release of rGO in PBS. Results 

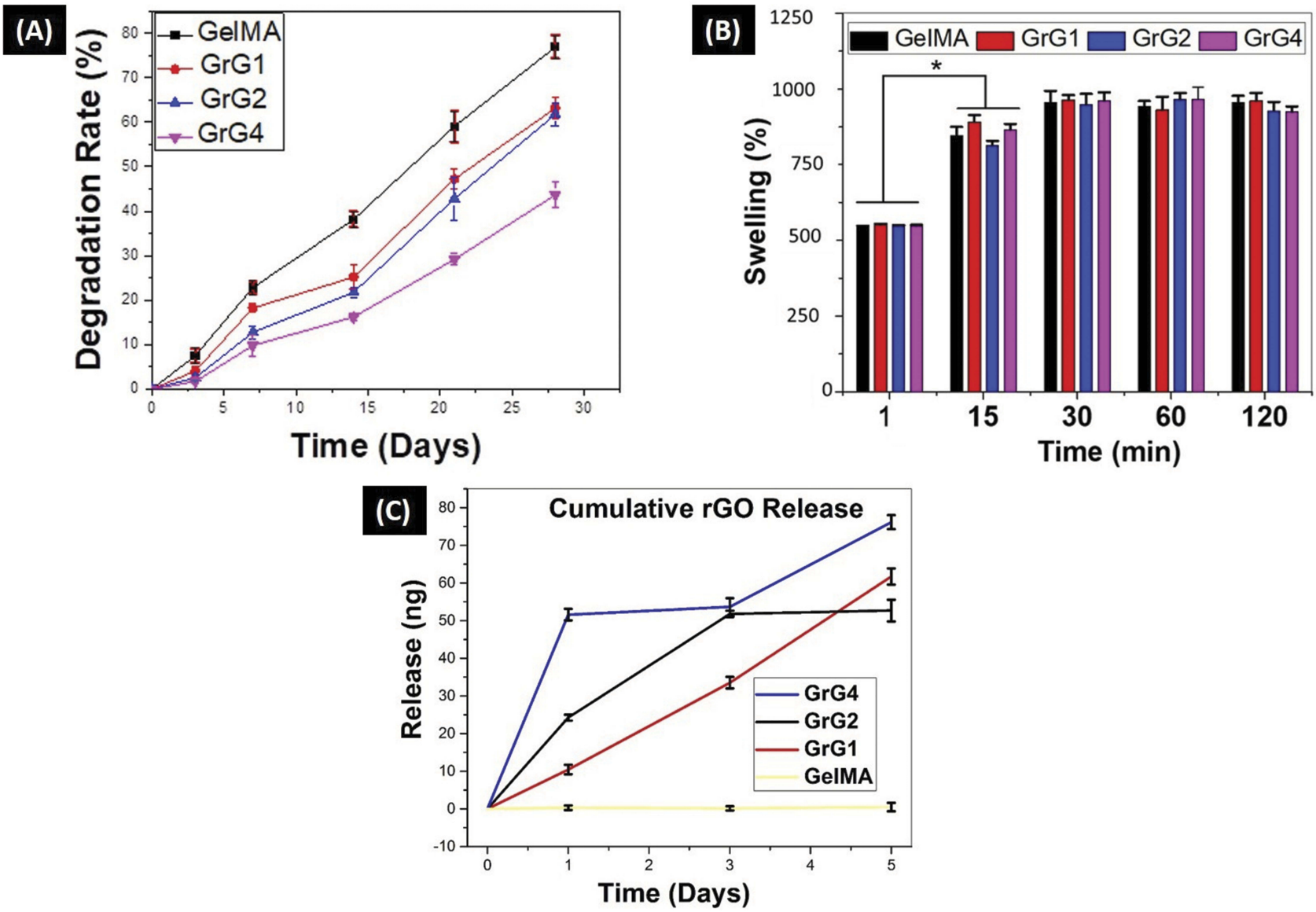

Figure 3 (A) Degradation curve of blank GelMA hydrogel and nanocomposite hydrogels with respect to time. Error bars represent the SD of measurements performed on at least 5 specimens. (B) The swelling percentage of blank GelMA hydrogel and nanocomposite GelMA hydrogel after $0,15,30,60$ and I20 mins respectively ( $* P<0.05)$. (C) Cumulative Release of rGO from the nanocomposite GelMA hydrogel (GrGI, GrG2, and GrG4) and blank GelMA hydrogel after I, 3 , and 5 days in PBS.

have shown a sustained and prolonged release of rGO from GelMA hydrogel. The release of rGO from GrG4 is comparatively higher than GrG1 and GrG2. However, both GrG1 and GrG2 has shown a uniform and steady release of rGO throughout the experiment. The swelling and degradation of GelMA hydrogel could be the reason of slow release of rGO from the hydrogel. Whereas, GrG4 showed burst release of rGO within 1 day and the release process was slowed down after 1 day. The initial burst release of rGO from GrG4 could be associated with the higher concentration of rGO present in the hydrogel. Such a higher release of nanoparticles from polymeric nanocomposite biomaterials is observed in other studies also. ${ }^{5}$ The traces of rGO was not observed in case of blank GelMA hydrogel, as expected. The slow release of rGO form GelMA hydrogel may stimulate the proliferation of the cells and eventually enhance the formation of new blood vessels.

\section{Biological Characterizations Live/Dead Assay}

Altough, rGO have potential beneficial effects on the biological system, some studies have also reported their adverse effects on cell viability. ${ }^{66}$ In order to visualize the cell viability by the distribution of live and dead cells after $24 \mathrm{hrs}$ of incubations, the Live/Dead cell assay on three different cell lines (3T3 fibroblasts, EA.hy926 endothelial cells, HaCat keratinocytes) was performed. The cell viability on culture media (control), blank GelMA hydrogel and nanocomposite hydrogels containing $0.001 \% \mathrm{w} / \mathrm{w}$ (GrG1), 0.002\% w/w (GrG2), and $0.004 \% \mathrm{w} / \mathrm{w}$ (GrG4) of rGO were evaluated and given in Figure 4A-O. The cells remained alive and no significant cytotoxic effect was observed in all samples up to $0.002 \% \mathrm{w} / \mathrm{w}$ concentration of $\mathrm{rGO}$. However, the percentage of dead cells was increased at higher concentration of rGO $(0.004 \% \mathrm{w} / \mathrm{w})$ as shown in Figure 4P. Overall results depict that the reduced graphene oxide up to $0.002 \% \mathrm{w} / \mathrm{w}$ concentration in GelMA hydrogel has almost no toxic effect on 


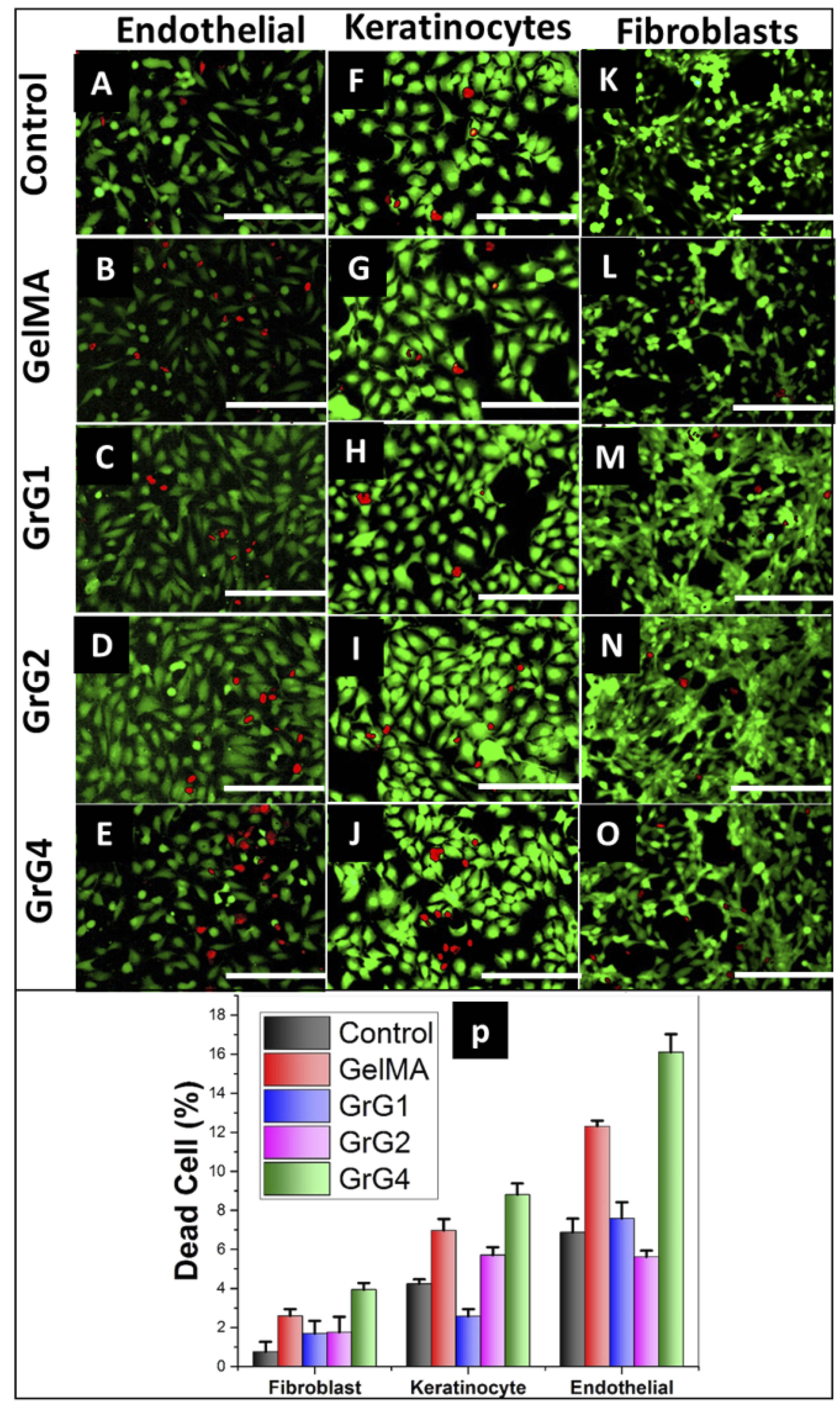

Figure 4 (A-E) Cell viability (Live/Dead assay) on Endothelial cells, (F-J) 3 T3 fibroblast cells and (K-O) HaCat keratinocyte cells for control, blank GelMA hydrogel, $0.00 \mathrm{I}$ wt\% rGO loaded GelMA hydrogel (GrGI), 0.002 wt\% rGO loaded GelMA hydrogel (GrG2) and 0.004 wt\% rGO loaded GelMA hydrogel (GrG4) respectively. Green channel depicts live cells, while red channels depict dead cells. (P) Quantitative comparison of the percentage of dead cells. The scale bar at the right lower corner is $1000 \mu \mathrm{m}$. 

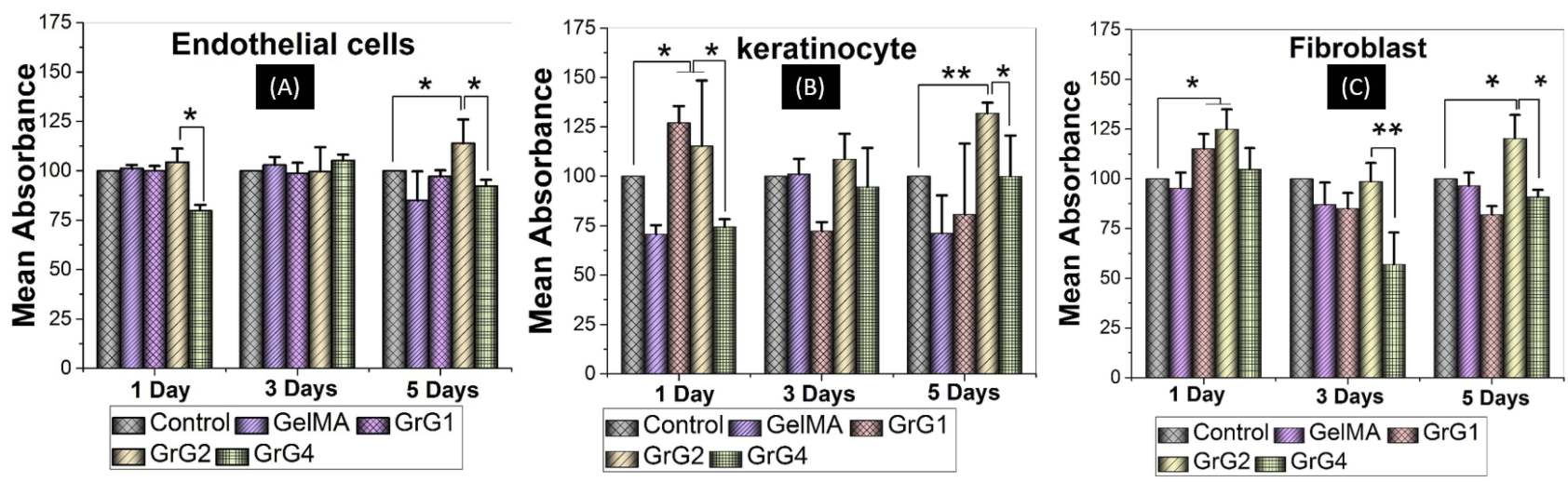

Figure 5 The histogram of MTT assay, comparing the proliferation of (A) endothelial cells, (B) 3T3 fibroblasts, and (C) HaCat keratinocytes after I day, 3 days, and 5 days of incubations. by the treatment of blank GelMA hydrogel, 0.001 wt\% rGO loaded GelMA hydrogel (GrGI), 0.002 wt $\%$ rGO loaded GelMA hydrogel (GrG2) and 0.004 wt\% rGO loaded GelMA hydrogel (GrG4) and control (untreated). (*P < 0.05, **P < 0.02).

studied cells. However, at higher concentration, the viability of the cells was significantly reduced. Furthermore, the morphology of the cells was not changed after the treatment of GelMA hydrogel and nanocomposite hydrogels.

The cell viability and cell attachment study of GelMA hydrogel and nanocomposite hydrogels have demonstrated the biocompatibility of the hydrogels. ${ }^{9}$ However, in this study, it has not shown any toxic effect at a concentration higher than its cytotoxic level as reported in the previous literature. ${ }^{59}$ The slow release of rGO from the hydrogel could be the reason for this observation where the cells might have exposed to a lower concentration than the cytotoxic level.

\section{MTT Assay}

In order to investigate the proliferation of $3 \mathrm{~T} 3$ fibroblasts, EA.hy926 endothelial cells, HaCat keratinocytes on GelMA hydrogel and nanocomposite hydrogels quantitatively, the MTT assay was implemented after 1, 3 and 5 days in culture. A significant effect of rGO concentration $\left({ }^{*} \mathrm{p}<0.05\right)$ on cell metabolic activity has demonstrated in Figure 5A-C. These results revealed all three cell lines has increased metabolic activity on rGO loaded GelMA hydrogel up to $0.002 \% \mathrm{w} / \mathrm{w}(\mathrm{GrG} 2)$ concentration compared to the control and blank GelMA hydrogel. However, increasing the concentration of $\mathrm{rGO}$ up to $0.004 \% \mathrm{w} / \mathrm{w}$ has reduced the proliferation of the cells. Particularly, after 1 day and 5 days in culture, MTT assay had shown the highest cellular metabolic activity of 3T3 fibroblasts and Hacat keratinocytes when $0.002 \%$ w/w rGO loaded GelMA hydrogel (GrG2) was used. The changing of the medium after 2 days of incubation, has reduced the amount to rGO in the medium. This might be the reason for less proliferation of cells in nanocomposite hydrogels compared to the controls at 3rd day of incubation. However, the release profile has shown that the rGO continues to release from the hydrogel after 3 days. Which proves that the new particles released from the hydrogel will again enhance the proliferation of the cells, as we can see in 5 days MTT assay results.

The MTT assay can measure the reduction of MTT dye depending upon the activity of mitochondrial dehydrogenase enzyme. Therefore, the higher metabolic measurement from the assay indicates the higher cell proliferation on the hydrogel surface. The release of rGO from the swollen or degrading hydrogels and its influence on cells could be the reason for the increased proliferation of the cells.

\section{Wound Healing Assay}

Wound healing assay or scratch assay is a standard in vitro technique for the cell migration study. ${ }^{67}$ In order to evaluate the potential of prepared nanocomposite hydrogels to enhance the migration of $3 \mathrm{~T} 3$ fibroblasts, EA.hy926 endothelial cells, and Hacat keratinocytes, a time-dependent experiment (0$24 \mathrm{hrs}$ ) was carried out, as shown in Figure 6A-C. In the case of fibroblasts, a significant wound healing $(* *=\mathrm{P}<0.01)$ was observed with the treatment of $0.001 \% \mathrm{w} / \mathrm{w}$ and $0.002 \%$ w/w rGO loaded GelMA hydrogel. Also, the endothelial cells and keratinocytes treated with $0.002 \% \mathrm{w} / \mathrm{w}$ rGO loaded GelMA hydrogel have migrated significantly $(*=\mathrm{P}<0.05)$ compared to the control (untreated) cells. However, at higher concentration, there is a significant reduction in cell migration $(* *=\mathrm{P}<0.01)$.The results depict that, GelMA hydrogel incorporated $0.002 \% \mathrm{w} / \mathrm{w}$ rGO (GrG2) induced the wound closure compared to control (untreated) and blank GelMA treated cells in all three cell lines (Figure 6A-C). However, rGO has not shown wound healing property (wound closure) 


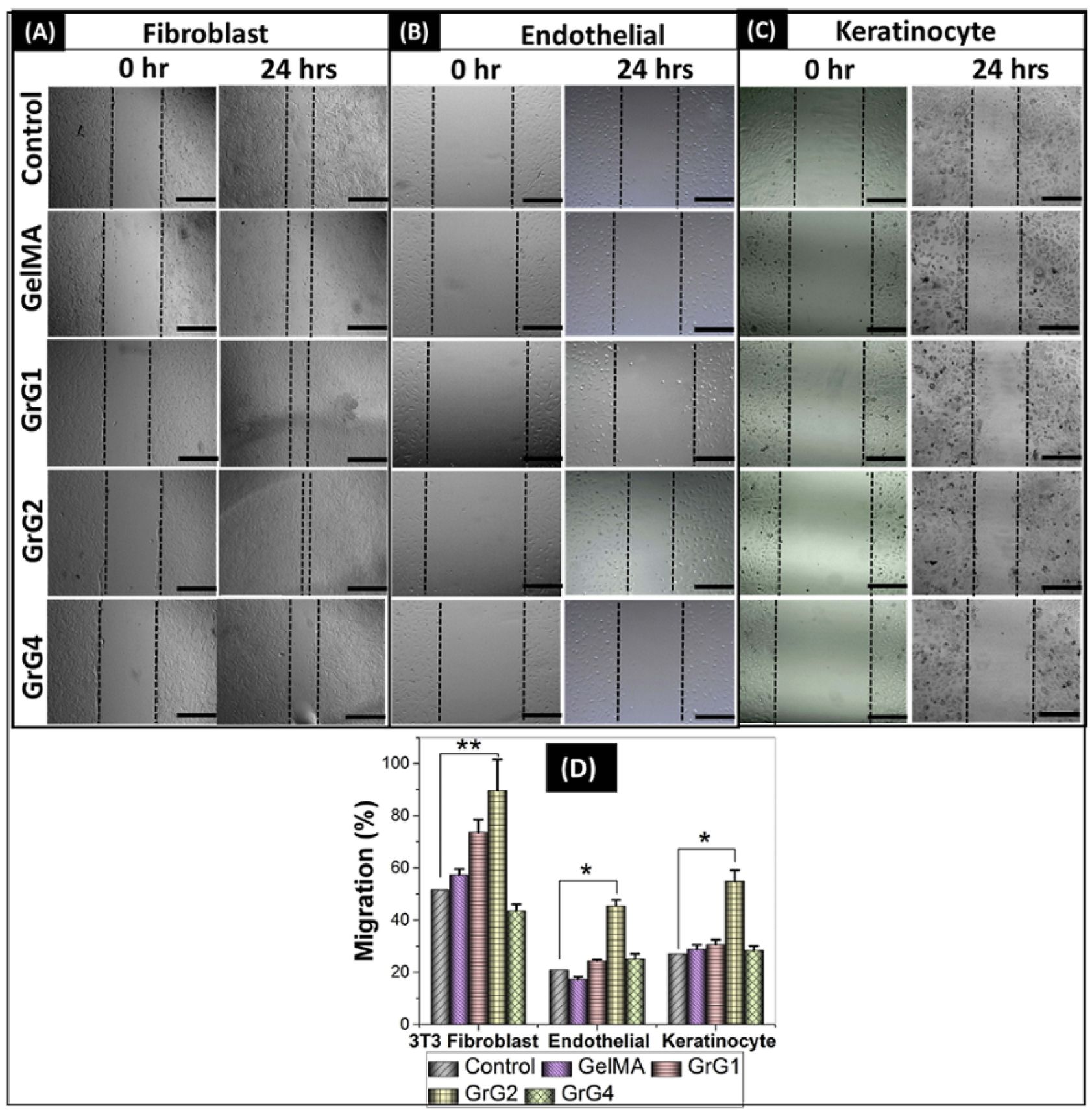

Figure 6 Results of wound healing scratch assay using (A) 3T3 fibroblast cells, (B) Endothelial Cells, (C) HaCat Keratinocyte cells for control (untreated), blank GelMA hydrogel, 0.001 wt\% rGO loaded GelMA hydrogel (GrGI), 0.002 wt\% rGO loaded GelMA hydrogel (GrG2), \& $0.004 \%$ rGO loaded GelMA hydrogel (GrG4) treated cells. (D) Percentage of wound healing was measured and presented on a histogram using Image software. ( $* \mathrm{P}<0.05, * * \mathrm{P}<0.0 \mathrm{I})$. The scale bar at the right lower corner is $\mathrm{I} 000 \mu \mathrm{m}$.

at higher concentration $(0.004 \% \mathrm{w} / \mathrm{w})$ compared to untreated control cells. Particularly, 3T3 fibroblast cells have shown maximum cell migration (85\%). In Figure 6D the extent of wound healing was quantified and presented in a histogram. The results altogether demonstrated that the cells at $0.002 \% \mathrm{w} /$ $\mathrm{w}$ concentration of rGO could enhance the migration of the cells which indicates their wound healing property.
The migration of native cells from the wound boundary is one of the key steps in the wound healing process. ${ }^{68}$ Recently, rGO has been reported to enhance the migration of endothelial cells at a concentration of $1-50 \mathrm{ng} \mathrm{mL}{ }^{-1}$ indicating their pro-angiogenic potential. ${ }^{9}$ Improved migration of fibroblasts in the wound area will play a key role in replacing the inflammatory cells (formed during the 


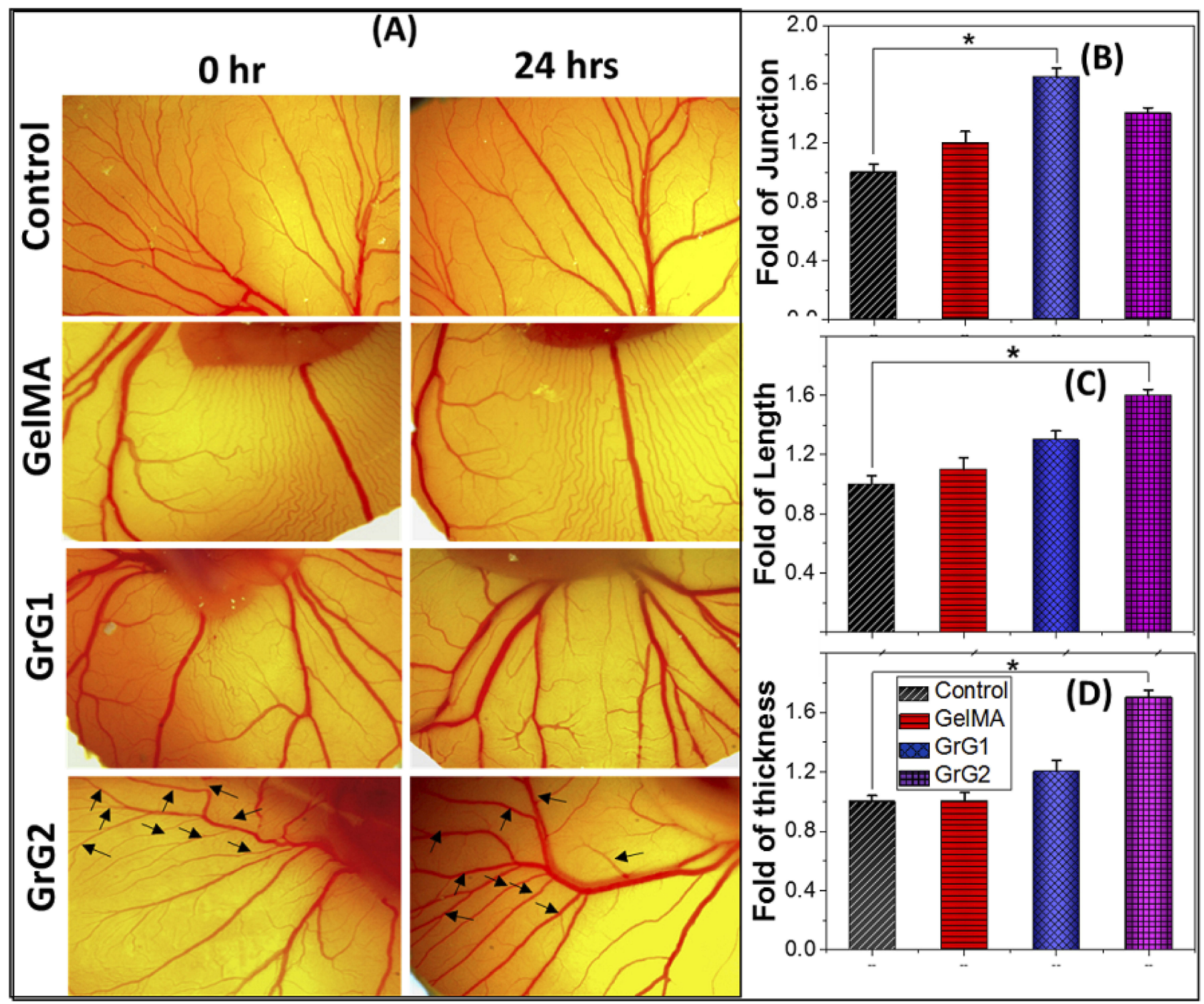

Figure 7 (A) Results of in vivo CEA assay of control (untreated samples), in the presence of the blank GelMA hydrogel, $0.00 \mathrm{I}$ wt\% rGO loaded GelMA hydrogel (GrGI), and 0.002 wt\% rGO loaded GelMA hydrogel (GrG2). Increase of matured blood vessel formation (marked by black arrows) was observed in embryo treated with GelMA hydrogel with 0.002 wt\% rGO nanoparticles (GrG2). Several angiogenic parameters such as blood vessel junction, length, and thickness were quantified and presented as a histogram (B-D respectively). Statistical significance was calculated by using $t$-test. All data are statistically significant $(* P<0.05)$.

inflammatory process) with collagen and native tissue. ${ }^{69}$ The endothelial cell migration will help in the formation of new blood vessels from the existing vasculature during angiogenesis. ${ }^{70}$ Furthermore, the migration of keratinocytes will enhance the re-epithelization process, which is critical and essential for successful wound healing. ${ }^{71}$ Thus, an optimal concentration of rGO in GelMA hydrogel could enhance the wound healing process by promoting the migration of different types of cells which are important in wound healing.

\section{In Vivo Chicken Embryo Angiogenesis (CEO) Study}

In order to evaluate the angiogenic potential of biomaterials, the chicken embryo angiogenesis (CEA) assay was used as a standard assay. It was carried out to understand the ability of the nanocomposite hydrogel to induce angiogenesis. The results of CEA assay are described in Figure 7A where the control, blank GelMA hydrogel, and $0.001 \%$ w/w (GrG1) and $0.002 \% \mathrm{w} / \mathrm{w}(\mathrm{GrG} 2)$ rGO loaded GelMA hydrogel were used. The number of branching points was also counted, change in length and thickness of blood vessels were measured using ImageJ software after $24 \mathrm{hrs}$ of incubation and presented in the histogram as shown in Figure 7B-D respectively. A significant increase $(*=\mathrm{P}<0.005)$ in matured vascular sprouting was observed in GelMA hydrogel incorporated with $0.001 \% \mathrm{w} / \mathrm{w}(\mathrm{GrG} 1)$, and $0.002 \% \mathrm{w} / \mathrm{w}$ (GrG1) rGO treated samples compared with the control (untreated) and blank GelMA hydrogel. The highest number of a 
matured blood vessels with highly branched capillary networks (1.7 times higher than the control) were observed at $0.001 \% \mathrm{w} / \mathrm{w}$ rGO loaded GelMA hydrogel (GrG1). The $0.002 \% \mathrm{w} / \mathrm{w}$ rGO loaded GelMA hydrogel (GrG2) treated sample has shown 1.6 and 1.7 times higher length and thickness of blood vessels, respectively than the control. The increase in vessel thickness is a sign of the maturation of nascent blood vessels and is possibly an outcome of superior angiogenesis. Thus, the obtained results suggested that the nanocomposite hydrogel has successfully enhanced the angiogenesis. The blank GelMA hydrogel treated samples did not show any significant angiogenic activity when compared with the control, as expected. Whereas, the untreated samples did not considerably increase the angiogenesis. Thus, with the increase of rGO concentration up to $0.002 \%$ $\mathrm{w} / \mathrm{w}$ in GelMA hydrogel the angiogenic potential of nanocomposite hydrogel was increased.

Earlier studies demonstrated that rGO has the ability to increase the intercellular concentration of ROS. ${ }^{9}$ The biomechanical machinery which is responsible for angiogenesis could be triggered by the transient increase of ROS concentration in the cells. However, further studies need to be performed to invariably confirm the molecular mechanism behind our observation.

\section{Conclusions}

In this study, we have successfully developed rGO incorporated GelMA hydrogel wound coverage materials with the pro-angiogenic property. Our prepared GelMA hydrogel displayed highly porous morphology. The XRD analysis confirmed the presence of rGO in GelMA hydrogels. In vitro cell viability assay (Live/Dead and MTT assay) demonstrated the biocompatibility of the hydrogel. Moreover, the developed material exhibited remarkable in vitro wound contraction potential in terms of improved fibroblast, keratinocytes, and endothelial cell proliferation. In addition, GelMA hydrogel containing $0.002 \% \mathrm{w} / \mathrm{w}$ rGO produced a large number of blood vessels with a highly branched capillary network in the chick embryo model compared to the blank GelMA hydrogel. We highly believe that our study on $\mathrm{rGO} / \mathrm{GelMA}$ hydrogel will put forward the insight for the advancement of angiogenic treatment strategies for several diseases where angiogenesis plays a significant role.

\section{Acknowledgments}

This article was made possible by the NPRP12S-0310190276 and NPRP9-144-3-021 grants funded by the
Qatar National Research Fund (a part of Qatar Foundation). We also acknowledge the support provided by the Central Laboratories Unit (CLU), Qatar University, Qatar. The publication of this article was funded by the Qatar National Library.

\section{Disclosure}

The authors declare no competing interests in this work.

\section{References}

1. Reiber GE, Boyko EJ, Smith DG. Lower extremity foot ulcers and amputations in diabetes. Diabetes Am. 1995;2:409-427.

2. Frykberg RG, Banks J. Challenges in the treatment of chronic wounds. Adv Wound Care. 2015;4(9):560-582. doi:10.1089/ wound.2015.0635

3. Zhao R, Liang $\mathrm{H}$, Clarke $\mathrm{E}$, et al. Inflammation in chronic wounds Int J Mol Sci. 2016;17(12):2085. doi:10.3390/ijms17122085

4. Ahmed R, Tariq M, Ali I, et al. Novel electrospun chitosan/polyvinyl alcohol/zinc oxide nanofibrous mats with antibacterial and antioxidant properties for diabetic wound healing. Int J Biol Macromol. 2018;120:385-393. doi:10.1016/j.ijbiomac.2018.08.057

5. Augustine R, Hasan A, Yadu Nath VK, et al. Electrospun polyvinyl alcohol membranes incorporated with green synthesized silver nanoparticles for wound dressing applications. J Mat Sci. 2018;29 (11): 163 .

6. Masood N, Ahmed R, Tariq M, et al. Silver nanoparticle impregnated chitosan-PEG hydrogel enhances wound healing in diabetes induced rabbits. Int J Pharm. 2019;559:23-36. doi:10.1016/j.ijpharm.2019.01.019

7. Mohammadi Z, Sharif Zak M, Majdi H, et al. The effect of chrysincurcumin-loaded nanofibres on the wound-healing process in male rats. Artif Cells Nanomed Biotechnol. 2019;47(1):1642-1652. doi:10.1080/ 21691401.2019.1594855

8. Wojtowicz AM, Oliveira S, Carlson MW, et al. The importance of both fibroblasts and keratinocytes in a bilayered living cellular construct used in wound healing. Wound Repair Regener. 2014;22 (2):246-255. doi:10.1111/wrr.12154

9. Mukherjee S, Sriram P, Barui AK, et al. Graphene oxides show angiogenic properties. Adv Healthc Mater. 2015;4(11):1722-1732. doi:10.1002/adhm.v4.11

10. Augustine R, Kumar Nethi SK, Kalarikkal N, et al. Electrospun polycaprolactone (PCL) scaffolds embedded with europium hydroxide nanorods (EHNs) with enhanced vascularization and cell proliferation for tissue engineering applications. J Mater Chem B. 2017;5(24):46604672. doi:10.1039/C7TB00518K

11. Shamloo A, Sarmadi M, Aghababaie Z, Vossoughi M. Accelerated full-thickness wound healing via sustained bFGF delivery based on a PVA/chitosan/gelatin hydrogel incorporating PCL microspheres. Int $J$ Pharm. 2018;537(1-2):278-289. doi:10.1016/j.ijpharm.2017.12.045

12. Sadr N, Zhu M, Osaki T, et al. SAM-based cell transfer to photopatterned hydrogels for microengineering vascular-like structures. Biomaterials. 2011;32(30):7479-7490. doi:10.1016/j.biomaterials.20 11.06 .034

13. Sorrell JM, Baber MA, Caplan AI. Influence of adult mesenchymal stem cells on in vitro vascular formation. Tissue Eng Part A. 2009;15 (7):1751-1761. doi:10.1089/ten.tea.2008.0254

14. Alajati A, Laib AM, Weber H, et al. Spheroid-based engineering of a human vasculature in mice. Nat Methods. 2008;5(5):439. doi:10.1038/ nmeth.1198

15. Lesman A, Habib M, Caspi O, et al. Transplantation of a tissueengineered human vascularized cardiac muscle. Tissue Eng Part A. 2009;16(1):115-125. doi:10.1089/ten.tea.2009.0130 
16. Saik JE, Gould DJ, Keswani AH, et al. Biomimetic hydrogels with immobilized ephrinA1 for therapeutic angiogenesis. Biomacromolecules. 2011;12(7):2715-2722. doi:10.1021/bm200492h

17. Mao AS, Mooney DJ. Regenerative medicine: current therapies and future directions. Proc Nat Acad Sci. 2015;112(47):14452-14459. doi:10.1073/pnas. 1508520112

18. Shah JB. The history of wound care. J Am Col Certif Wound Spec. 2011;3(3):65-66. doi:10.1016/j.jcws.2012.04.002

19. Augustine R, Kalarikkal N, Thomas S. Advancement of wound care from grafts to bioengineered smart skin substitutes. Progress Biomater. 2014;3(2-4):103-113. doi:10.1007/s40204-014-0030-y

20. Balakrishnan B, MOHANTY M, UMASHANKAR P, et al. Evaluation of an in situ forming hydrogel wound dressing based on oxidized alginate and gelatin. Biomaterials. 2005;26(32):6335-6342. doi:10.1016/j.biomaterials.2005.04.012

21. Kennedy JF, Knill CJ, Thorley M. Natural polymers for healing wounds. In: Kennedy JF, Phillips GO, Williams PA, editors. Recent Advances in Environmentally Compatible Polymers. Elsevier; 2001:97-104.

22. Augustine R, Augustine A, Kalarikkal N, et al. Fabrication and characterization of biosilver nanoparticles loaded calcium pectinate nano-micro dual-porous antibacterial wound dressings. Progress Biomater. 2016;5(3-4):223-235. doi:10.1007/s40204-016-0060-8

23. Farhat W, Hasan A, Lucia L, Becquart F, Ayoub A, Kobeissy F. Hydrogels for advanced stem cell therapies: a biomimetic materials approach for enhancing natural tissue function. IEEE Rev Biomed Eng. 2018.

24. Slaughter BV, Khurshid SS, Fisher OZ, et al. Hydrogels in regenerative medicine. Adv Mater. 2009;21(32-33):3307-3329. doi:10.1002/ adma.v21:32/33

25. Wickremasinghe NC, Kumar VA, Hartgerink JD. Two-step self-assembly of liposome-multidomain peptide nanofiber hydrogel for time-controlled release. Biomacromolecules. 2014;15(10):3587-3595. doi:10.10 21/bm500856c

26. Biondi M, Ungaro F, Quaglia F, et al. Controlled drug delivery in tissue engineering. Adv Drug Deliv Rev. 2008;60(2):229-242. doi:10.1016/j. addr.2007.08.038

27. Vashist A, Vashist A, Gupta YK, et al. Recent advances in hydrogel based drug delivery systems for the human body. J Mater Chem B. 2014;2(2):147-166. doi:10.1039/C3TB21016B

28. Biondi M, Indolfi L, Ungaro F, et al. Bioactivated collagen-based scaffolds embedding protein-releasing biodegradable microspheres: tuning of protein release kinetics. J Mat Sci. 2009;20(10):2117-2128. doi:10.1007/s10856-009-3766-5

29. Mollica F, Biondi M, Muzzi S, et al. Mathematical modelling of the evolution of protein distribution within single PLGA microspheres: prediction of local concentration profiles and release kinetics. J Mat Sci. 2008;19(4):1587-1593. doi:10.1007/s10856-007-3301-5

30. Ungaro F, Biondi M, d'Angelo I, et al. Microsphere-integrated collagen scaffolds for tissue engineering: effect of microsphere formulation and scaffold properties on protein release kinetics. $J$ Controlled Release. 2006;113(2):128-136. doi:10.1016/j.jconrel.2006.04.011

31. Mayol L, Biondi M, Russo L, et al. Amphiphilic hyaluronic acid derivatives toward the design of micelles for the sustained delivery of hydrophobic drugs. Carbohydr Polym. 2014;102:110-116. doi:10.10 16/j.carbpol.2013.11.003

32. Maltese A, Borzacchiello A, Mayol L, et al. Novel polysaccharidesbased viscoelastic formulations for ophthalmic surgery: rheological characterization. Biomaterials. 2006;27(29):5134-5142. doi:10.1016/ j.biomaterials.2006.05.036

33. Paul A, Hasan A, Kindi HA, et al. Injectable graphene oxide/hydrogelbased angiogenic gene delivery system for vasculogenesis and cardiac repair. ACS Nano. 2014;8(8):8050-8062. doi:10.1021/nn5020787

34. Paul A, Manoharan V, Krafft D, et al. Nanoengineered biomimetic hydrogels for guiding human stem cell osteogenesis in three dimensional microenvironments. J Mater Chem B. 2016;4(20):3544-3554. doi:10.1039/C5TB02745D
35. Zhang R, Tao Y, Xu W, et al. Rheological and controlled release properties of hydrogels based on mushroom hyperbranched polysaccharide and xanthan gum. Int J Biol Macromol. 2018;120:2399-2409. doi:10.1016/j.ijbiomac.2018.09.008

36. Liang Y, Zhao X, Hu T, et al. Adhesive hemostatic conducting injectable composite hydrogels with sustained drug release and photothermal antibacterial activity to promote full-thickness skin regeneration during wound healing. Small. 2019;15(12):1900046. doi:10.1002/smll.v15.12

37. Liang Y, Zhao X, Ma PX, et al. pH-responsive injectable hydrogels with mucosal adhesiveness based on chitosan-grafted-dihydrocaffeic acid and oxidized pullulan for localized drug delivery. J Colloid Interface Sci. 2019;536:224-234. doi:10.1016/j.jcis.2018.10.056

38. Zhao X, Li P, Guo B, et al. Antibacterial and conductive injectable hydrogels based on quaternized chitosan-graft-polyaniline/oxidized dextran for tissue engineering. Acta Biomater. 2015;26:236-248. doi:10.1016/j.actbio.2015.08.006

39. Majumder P, Baxa U, Walsh STR, et al. Design of a multicompartment hydrogel that facilitates time-resolved delivery of combination therapy and synergized killing of glioblastoma. Angewandte Chemie. 2018;130(46):15260-15264. doi:10.1002/ange.v130.46

40. Ramón-Azcón J, Ahadian S, Obregón R, et al. Gelatin methacrylate as a promising hydrogel for $3 \mathrm{D}$ microscale organization and proliferation of dielectrophoretically patterned cells. Lab Chip. 2012;12 (16):2959-2969. doi:10.1039/c2lc40213k

41. Cheng H, Yue K, Kazemzadeh-Narbat M, et al. Mussel-inspired multifunctional hydrogel coating for prevention of infections and enhanced osteogenesis. ACS Appl Mater Interfaces. 2017;9 (13):11428-11439. doi:10.1021/acsami.6b16779

42. Chen H, Guo L, Wicks J, et al. Quickly promoting angiogenesis by using a DFO-loaded photo-crosslinked gelatin hydrogel for diabetic skin regeneration. J Mater Chem B. 2016;4(21):3770-3781. doi:10.1039/ C6TB00065G

43. Gkikas M, Avery RK, Olsen BD. Thermoresponsive and Mechanical Properties of Poly. American Chemical Society; 2016.

44. Pepelanova I, Kruppa K, Scheper T, et al. Gelatin-Methacryloyl (GelMA) hydrogels with defined degree of functionalization as a versatile toolkit for 3D cell culture and extrusion bioprinting. Bioengineering. 2018;5(3):55. doi:10.3390/bioengineering5030055

45. Sun M, Sun X, Wang Z, et al. Synthesis and properties of Gelatin Methacryloyl (GelMA) hydrogels and their recent applications in loadbearing tissue. Polymers. 2018;10(11):1290. doi:10.3390/polym10111290

46. Augustine R, Hasan A, Patan NK, et al. Cerium oxide nanoparticle incorporated electrospun poly (3-hydroxybutyrate-co-3-hydroxyvalerate) membranes for diabetic wound healing applications. ACS Biomater Sci Eng. 2019. doi:10.1021/acsbiomaterials.8b01352

47. Augustine R, Kalarikkal N, Thomas S. Electrospun PCL membranes incorporated with biosynthesized silver nanoparticles as antibacterial wound dressings. Appl Nanosci. 2016;6(3):337-344. doi:10.1007/ s13204-015-0439-1

48. Terzopoulou Z, Kyzas G, Bikiaris D. Recent advances in nanocomposite materials of graphene derivatives with polysaccharides. Materials. 2015;8(2):652-683. doi:10.3390/ma8020652

49. Palejwala AH, Fridley JS, Mata JA, et al. Biocompatibility of reduced graphene oxide nanoscaffolds following acute spinal cord injury in rats. Surg Neurol Int. 2016;7.

50. Huang Y, Zeng M, Chen J, et al. Multi-structural network design and mechanical properties of graphene oxide filled chitosan-based hydrogel nanocomposites. Mater Des. 2018;148:104-114. doi:10.1016/j. matdes.2018.03.055

51. Räder HJ, Rouhanipour A, Talarico AM, et al. Processing of giant graphene molecules by soft-landing mass spectrometry. Nat Mater. 2006;5(4):276. doi:10.1038/nmat1597

52. Wujcik EK, Monty CN. Nanotechnology for implantable sensors: carbon nanotubes and graphene in medicine. Wiley Interdiscip Rev. 2013;5(3):233-249. doi:10.1002/wnan.1213 
53. Wang C, Li J, Amatore C, et al. Gold nanoclusters and graphene nanocomposites for drug delivery and imaging of cancer cells. Angewandte Chemie Int Ed. 2011;50(49):11644-11648. doi:10.1002/anie.201105573

54. Garaj S, Hubbard W, Reina A, et al. Graphene as a subnanometre trans-electrode membrane. Nature. 2010;467(7312):190. doi:10.1038/ nature 09379

55. Pyun J. Graphene oxide as catalyst: application of carbon materials beyond nanotechnology. Angewandte Chemie Int Ed. 2011;50(1):4648. doi:10.1002/anie.v50.1

56. Tang J, Chen Q, Xu L, et al. Graphene oxide-silver nanocomposite as a highly effective antibacterial agent with species-specific mechanisms. ACS Appl Mater Interfaces. 2013;5(9):3867-3874. doi:10.1021/ am4005495

57. Ushio-Fukai M, Alexander RW. Reactive oxygen species as mediators of angiogenesis signaling. Role of NAD (P) H oxidase. Mol Cell Biochem. 2004;264(1-2):85-97. doi:10.1023/B:MCBI.0000044378.09409.b5

58. Augustine R, Dan P, Sosnik A, et al. Electrospun poly (vinylidene fluoride-trifluoroethylene)/zinc oxide nanocomposite tissue engineering scaffolds with enhanced cell adhesion and blood vessel formation. Nano Res. 2017;10(10):3358-3376. doi:10.1007/s12274-017-1549-8

59. Chakraborty S, Ponrasu T, Chandel S, et al. Reduced graphene oxideloaded nanocomposite scaffolds for enhancing angiogenesis in tissue engineering applications. $R$ Soc Open Sci. 2018;5(5):172017. doi:10. 1098/rsos. 172017

60. Augustine R, Hasan A, Patan NK. et al. Titanium nanorods loaded PCL meshes with enhanced blood vessel formation and cell migration for wound dressing applications. Macromol Biosci;2019. 1900058. doi:10.1002/mabi.201900058

61. Liu J, Zheng X, Tang K. Study on the gravimetric measurement of the swelling behaviors of polymer films. Rev Adv Mater Sci. 2013;33 (5):452-458.
62. Zhou L, Tan G, Tan Y, et al. Biomimetic mineralization of anionic gelatin hydrogels: effect of degree of methacrylation. RSC Adv. 2014;4(42):21997-22008. doi:10.1039/C4RA02271H

63. Sahoo P, Panigrahy B, Li D, et al. Magnetic behavior of reduced graphene oxide/metal nanocomposites. J Appl Phys. 2013;113(17):17B525. doi:10. $1063 / 1.4799150$

64. Si S, Zhou R, Xing Z, et al. A study of hybrid organic/inorganic hydrogel films based on in situ-generated $\mathrm{TiO} 2$ nanoparticles and methacrylated gelatin. Fibers Polym. 2013;14(6):982-989. doi:10.1007/s12221-0130982-x

65. Zahid AA, Ahmed R, ur Rehman, Augustine R, Tariq M, Hasan A. Nitric oxide releasing chitosan-poly (vinyl alcohol) hydrogel promotes angiogenesis in chick embryo model. Int $\mathrm{J}$ Biol Macromol. 2019;136:901-910. doi:10.1016/j.ijbiomac.2019.06.136

66. Sahu SC, Hayes AW. Toxicity of nanomaterials found in human environment: a literature review. Toxicol Res Appl. 2017;1:2397847317726352. doi:10.1177/2397847317726352

67. Ilina O, Friedl P. Mechanisms of collective cell migration at a glance. J Cell Sci. 2009;122(18):3203-3208. doi:10.1242/jcs.036525

68. Velnar T, Bailey T, Smrkolj V. The wound healing process: an overview of the cellular and molecular mechanisms. J Int Med Res. 2009;37(5):1528-1542. doi:10.1177/147323000903700531

69. McDougall S, Dallon J, Sherratt J, et al. Fibroblast migration and collagen deposition during dermal wound healing: mathematical modelling and clinical implications. Philos Trans $R$ Soc $A$. 2006;364(1843):1385-1405. doi:10.1098/rsta.2006.1773

70. Eilken HM, Adams RH. Dynamics of endothelial cell behavior in sprouting angiogenesis. Curr Opin Cell Biol. 2010; 22(5):617-625. doi:10.1016/j.ceb.2010.08.010

71. Raja SK, Garcia MS, Isseroff RR. Wound re-epithelialization: modulating keratinocyte migration in wound healing. Front Biosci. 2007;12(3):2849-2868. doi:10.2741/2277
International Journal of Nanomedicine

\section{Publish your work in this journal}

The International Journal of Nanomedicine is an international, peerreviewed journal focusing on the application of nanotechnology in diagnostics, therapeutics, and drug delivery systems throughout the biomedical field. This journal is indexed on PubMed Central, MedLine, CAS, SciSearch ${ }^{\circledR}$, Current Contents ${ }^{\mathbb{R}} /$ Clinical Medicine,
Journal Citation Reports/Science Edition, EMBase, Scopus and the Elsevier Bibliographic databases. The manuscript management system is completely online and includes a very quick and fair peer-review system, which is all easy to use. Visit http://www.dovepress.com/ testimonials.php to read real quotes from published authors. 